SUBJECT INDEX

Note: Most organisations, serials and projects are listed in this index under their acronyms or abbreviations (see Appendix 1).

A\&A Monthly Index $11,12,85,181$

AAA $9,12,17,18,38,66-8,77-81,84$, $87-8,92,93,129,130,133,151,200$

AACR2 17,129

AAO $23,28,74,188,197$

AAS $6,38,45-7$

AAVSO $16,18,30,222-4$

Abbreviations 9, 43, 231-3

Abstracting 8,9,77-9,82,85

Acquisitions $7-9,15,48-57,128,217$

Acronyms 97, 231-3

ADC 19,124-5

Addresses $14,17,97,103,177,198$, 234-240

Administration 25-6,190-1

See also Management

AIP $6,11,12,82-3$

Annual reports $17,18,129$

ANSI 29,76

Archiving 22-5, 30,157-160, 227

correspondence 168-170

data 19-21,124-5,139-145

documents $24,164-170$

ARI $12,77-8,84$

Arrangement 129,134

Artificial intelligence $31,180,204-5$

Astronomers $24,25-6,27,28,30,97$

Astronomical Almanac 27,135

Astrophysical Journal 5,6,46,86

Authors 5, 6, 7, 42-3, 215

Beijing Observatory 57,58

Bibliographies 29,79,84-90,129,130, 150-3, 213

Binding 132,157-160

Books 14, 29, 44,226

care of $22-3,157-160$

catalogs $52,93-6$

rare $21,22,29,157-160,226$

Boxes $22,23,24,132$

British Library 29

Browsing 14

Budgets 37,190

Bulletin Board 15
Card catalogs $27,74,122,134,136,179$, 188,227

Catalogs

data $7,9,11,16-7,19,51,84,117$, $123-6,147-8,213$

library 27, 179-180,184,186,196, 227 See also Card catalogs observations 19-21,139-144

Cataloging 18,127,129,132,182

CD-ROM 19, 26-7, 39

CDS $12,13,16,18,19,84,97-8,146$, 149,150

China $8,18,26,28,30,56-61,136,182-3$, 199-202, 219-221

Chinese language $8,57,61,221$

Citations 47,85,87

Classification $7,9,10,11,14,17,32,66$, $70-1,75,79,83,87-8,90,129-130,134$ CODATA 17,19

Compact disks $19,26-7,39,43,118$

Computers, use of $10,15,26,31$, 99-101,175-183, 205-206, 227

See also Networks

Conference proceedings 55,84

Conservation 22-25,32,157-160

Contents 8,16

Cooperation 27-8,30,184-7,192-3, 211-3

Copyright $16,29,31$

Correspondence 164-170

Costs 5-7,31,39-42,45-7

CSIRO $28,196-7$

Currency $7,8,49,54,127,194$

Current Contents 85,110

DAO 16

Data archives 19-21,124-5,139-145

Data centres $8,19-22,43,125$

Data management $19-21,123-5$

Data services 19-22

Databases 21

astronomical (see numerical)

bibliographic $10,12,31,74,84-90$, 113-6,150-3,175-183,227

numerical $17,89-90,91,146-9,222-4$, 228 
DAVID 21

Descriptors $11,12,67-9,82$

Designations $12,21,67,88,140,142$, 144,147-8

Developing countries 8,28

Directories $13,19,45,97-8,103,193$

Disaster plans 23

Discs: see Disks

Diskettes $13,15,26,43,94,117$

Disks

compact $19,26,39,43,118$

floppy (see diskettes)

optical $20,21-2,25,118,124,140,144$

Display 134

Distribution $18,28,32,127,135,228$

Documentation 16,30,215-6

Documents 22-5,32,162,164-7

Duplicates 28,30,135,192,194-5

Editors 5, 42-3,

Education 30,45

Electronic mail $4,13,14,15,28,30,31$, $32,43,97,102-4,112,149,181,191,198$

E-mail: See Electronic mail

English language 8

Environment 21,31,136,158-160

Ephemerides 135,167

ESA $91,145,194$

ESIS 12,91-2

ESO 20,111-2,122,141-4,175-8

ESOC 21

Exchange 8,14,17,28,30,49-51,53,55, $56-7,132,192,194-5,199-202,226$

Exhibitions 22,157-160

Films 19

FID 701

First Dictionary 74,147

FITS format $20,139,141-2,144$

FIZ $12,78-9,84,150-1$

French libraries 217-8

Glass plates 119-121

Grey literature 214

GSFC 3,5,18-19,21,145

Historical records 24-25,164-172, 227

History $32,211-326,30$,

\section{IAU 38,213}

Circulars 14, 181

Colloquium 110: 3-34

Commission 5 : $3,4,7,9,11,25,28$, 29-30,32, 69, 71,74-5, 225

Commission 41: 25,30,32

Executive Committee 5,29
IAU continued

General Assemblies 3,5,11,22,29, 33

Information Bulletin $\mathbf{8 , 5 3}$

Joint Discussion 3-4,33

Style Manual 9,29

Telegram Bureau 14

Thesaurus $10,11,32,72-6$

IFLA 7

Indexing 9-12,65-9

India 109

Information

exchange $28,32,53,192$

services $219-221$

sharing 28,198

See $n^{\prime}$ so Retrieval

INSPEC $12,17,30,85,89-90,92$

Instruments $23,24,32,161-3,170,227$

ISO 20,29

ISSN 13,97

IUE $20,21,145,152$

Journals 5-6,8,15,17, 37-43, 45-7, $58-9,82,84,104,226$

J. RAS Canada 17

Keywords 9,12,67-8,79,82,87,139,150

Kluwer Publ. Co. 5,6,16

Konkoly Obs. 161-2,227

La Palma 19,21,139-140

La Silla 14,111-2,122,175

Languages 26,177

LANs $99-100$

LATEX $26,175,177,181$

LC classification $7,9,10,129-130,135$

Letters $25,177,181$

Librarians $13,17,24,25-6,27,30,31$, $93,175,207-8,227$

Libraries $13,25-31,32,48-51,76,127-8$, $175-208,225-8$

Library of Congress $4,9,21,22,29$, 157-8

Lists $26,177,181,194-5$

See also Union lists

Literature, astron. 5,37-44,48-51, $56-61$

Loans 184-5

Magnetic tape $11,12,16,18,19,20$, $117-8,124-5,136,140,142,228$

Management $18,25-6,136,169,182-3$, $186,188,190-1$

Mass-storage devices 20,124

Mathematics 217-8

MATHOR 43

Microfiche 13,16,18,19,26, 27, 43,96, $117,122,136,180,196,227$ 
Microfilm 25, 40, 227

Monthly Index $11,12,85,181$

Mount Wilson Obs. 17,134

NASA $19,29,149,194$

Names $20,129-130,140,147-8,150$

Networks $12,13,19,21,26-7,99-101$, $102,125,196,227$

Newsletters $19,30,45,129,131,133$, $152,224,225$

NOAO 14-5

Non-printed materials $15-6,117-8,136$

NRAO 15,113-6,195

NSSDC $18-19,22$

Objects, astron. $12,21,84,87,90,140$, 146-153

Observations 19-20,139-144,222-3

Observatories 97,129-130

Observatory publications 17-8,26,32, $60-1,80,96,127-136,175,184,212$

On-line access $12,13,17,19,20,43$, $109,113-6,175,179,185,188,196$, 207,227

Optical disks 20,21-2,25,118,124,140, 144,228

PACS $9,10,12,75,82-3,149$

Packet switching $13,19,21,101,175$

Page charges 5,6,7,41-2, 47

PAM $18,28,30,31,32,93,192$

Papers personal 24,164-170

published 6,37-39

Paris Obs. 24,164-5,170

Peculiarities 18

Periodicals 26, 55, 176-8, 212

Photocopying 21

Photographs $16,22,23,24,32,119-121$

PHYS $12,80,82,84,87-8$

Physics 82, 217-8

Physics Briefs $12,82,84$

Plates 16,119-121,227

Preprints 14-15,17,18, 25,32,109-116, $129,131-2,134,136,175,181,201$

Prices 5-6

Printing 212

Proceedings 55,84

PSS 13,19,21,101,175

Publications 30, 31, 58-61, 129

See also Observatory publications

Publishing 5-7, 37-47,66,82, 207, 227

Purple Mountain Obs. 56,58,60-1 199-202

Questionnaire 225-8
Radcliffe Obs. 23

Radio astronomy $10,14,109,113,196$

Rare books 21,22, 29,157-160,226

Records, historical 24-25,164-172

Refereeing 6,42-3,83

References $21,150-3$

Remote sites $27,190-1,197,226$

Reports 129,132

Reprints $14,17,18,130,131,136$

Research $31,45,211,214$

Resource sharing 27-9,192-8

Retrieval $9-14,22,31,43,65-105,125$, 146-8, 180-3

Reviewing 42

RGO $3,19,22,23,26,95,139-140$

Royal Obs. Belgium 184-6

Royal Obs. Edinburgh 95-6

Royalties 5

Russian language 8,80

SAO $11,18,30,215-6$

Searching 9-14,65-105

See also Retrieval

Selection 24,169

Serials $11,13,17,28,32,93-6,199-202$

Shaanxi Ast. Obs. 26,58,60-1,182-3, 221

Shanghai Obs. 56,58,60-1

Silver fish 23

SIMBAD $12,20-1,22,84,98,125,144$, $146-153$

Sky surveys $15,119-121,136,190$

SLA $28,30,31,32,93,192$

Slide collections 16

Smithsonian Inst. 23

Societies, scientific 45-7,97

Softstrip 16, 30

Software $14,19,26,30,43,95,104$, $139,181,185-6,188,215-6$

Space, storage $37-9,133,140$

Spacecraft data $21,85,140,145$

SPAN $20,21,22,86,102,125,175$

Special-format materials 14-8,136

SPIN 82,85

Staff $131,190,227$

Standards 29

Stocktaking 27

Storage $37-40,119-121,133,140$, $157-160,179-181$

STScI 15, 113-6,175,195

Subject headings 10

Subscriptions $8,14,17,18,40-2,45-7$, $49,55,176$

Surveys $19-20,225-8$ 
Technology 42-4,170,191, 203-4

Telefax 4,28,185,190,198

Telephone systems $13,19,21$

Telescopes $19,20,139-140$

Terminals $27,109,180$

TEX $43,103,181$

Thesaurus $10,11,32,67-9,72-6,90,150$

Theses 18

Third World Academy 28

Titles 8,11,17,130,134,176,215

Toronto Univ. 30,129

Translations $9,17,61,82,129,192$

Transliteration 129,130

Transport 17,190

UDC $9,10,32,70-1$

UNESCO 11

Union lists $13,28,32,93-6,132,196$

USA 29,192

USNO $3,4,23,131$

USSR $8,9,18,29,48-53$

Variable stars $7,9,51,90,152-3,222-4$

Video tapes $20,27,142$

Visitors 190

Vocabulary $1,9,168,72$

WANs 13,99

Warsaw Obs. 127

Water damage 23-4,159

Word processors 43

X25: 13,144 